\title{
Blockchain: Technological Revolution in Business and Administration
}

\author{
Ehab Khalifa \\ Future Center for Advanced Research and Studies
}

Blockchain is the Technology which allows assets to be transferred from one party to another simultaneously and without needing a third party, guaranteeing utmost security. In addition, it is a multiple global ledgers, shared by all people around the world, which enables them to do all kinds of transactions (Sutardja Center for Entrepreneurship \& Technology Technical Report, 2015) in real time and without falling prey to fraud or manipulation. Besides, it is the biggest data bases, accessible to all people (BigchainDB, 2016) and containing verified assets which can be safely exchanged.

Blockchain will have a huge effect on deferent levels, it will change the way all people adopt to deal through decades and threat traditional intermediary parties, on the other side it faces many obstacles stumbling blocks to be fully operated in different fields. This paper try to address the mechanism of blockchain technology, and how it works in simple words, then determine the possible applications of blockchain, and how to make best use of this technology, and finally the threats and challenges of blockchain.

\section{WHY BLOCKCHAIN IS A REVOLUTION?}

The blockchain system was first used in 2008 as the main platform for Bitcoin's virtual currency. Thanks to Blockchain, this cryptocurrency has won users' trust, so far at least. It is a common misconception that Bitcoin and Blockchain are one and the same; the truth is that the latter can be regarded as the backbone of the former, which distinguishes it from other digital currency systems (KAYE Scholar, 2018). It is worth mentioning that the Blockchain system can be used in many other applications.

Traditional transactions and asset transfer usually involve mediators. These can be banks, necessary for money transfer, a ministry entitled to register transactions, a real estate publicity department to verify ownership, a real estate broker, etc. These intermediary entities are usually paid for their services. Blockchain provides an alternative where files are transferred, stored and managed with no need for such mediators. To be more accurate, the real mediator in this case would be millions of computers connected to the chain. The transaction moves from one computer to another in an encrypted form that guarantees safety, rules out the possibility of manipulation, forgery or fraud and secures registration priority rights. This poses a threat to millions of people around the world who would be more than likely to lose their jobs because of this system. 
As a matter of fact, what makes Blockchain one of the driving forces of the smart revolution which humanity is witnessing, as well as one of the most important tools that would help people manage their life better, is the following:

1. The ability to transfer original files: The typical situation when transferring files is that only copies of these files are transferred. For instance, when an email, or a file, is sent via the internet, the data sent can be retained by the sender, as what is sent is actually a copy, not an original. This is undoubtedly not suitable when the thing to be transferred is money; you simply cannot send one hundred dollars to someone and keep them for yourself at the same time. This also applies to voting, intellectual property rights, patents and to cases where one buys the original files of songs and movies so that no other party can have access to them (Tapscott, 2018).

2. Immunity to manipulation: Perhaps more important is Blockchain's immunity to manipulation. Blockchain prevents fraud during and after transactions. It guarantees the security of such processes as transferring money, parcels, containers, etc., registering contracts or property, shipping goods, routing and doing government transactions. Depending on Blockchain prevents any attempt of manipulation that would constitute a threat to the country's financial resources or result in denying certain parties their right to a level playing field. Thus, depending on Blockchain would play an essential role in combating corruption as it ensures that transactions will not be altered, modified or deleted later. This would help create an atmosphere of confidence among users (Sutardja Center for Entrepreneurship \& Technology Technical Report, 2015).

\section{HOW DOES BLOCKCHAIN WORK?}

Blockchain functions according to three operating principles that together constitute the basis underlying the system and govern all transactions among users. These are:

1. The open ledger principle:

This refers to the accessibility of the information in Blockchain to everyone. In other words, everyone on a certain chain would be able to see everyone else's assets. For instance, if the chain is a money transfer chain everyone on it can see the others' money. However, people can choose not to reveal their identities, as Blockchain makes it possible for them to use nicknames suggested by the chain. Thus, while it would be difficult to get information about others' identities, information about their assets would be available to all users with utmost transparency (Iansiti and Lakhani, 2017).

Perhaps an example would be useful here. If (A) wishes to transfer ten dollars to (B), all the chain users will be able to know whether (B) has ten dollars. In case $\mathrm{s} /$ he does not, the transaction will not be completed as people would refrain from responding. If $\mathrm{s} / \mathrm{he}$ does have the money then the person nearest him, say (C), will give (B) the money in return for a percentage of the money that (A) wants to transfer.

One disadvantage to this operating principle is that it makes it possible for people to know personal information about others on the chain. Having access to a user's ledger makes it possible to know, for example, that he is a family man and that he transfers money to his family members (wife, children, etc.). It also makes it possible for people to know how much money he has and when exactly he transfers money to his wife, etc. Such information can be utilized in criminal activities targeting this user's family.

2. Distributed database:

The main objective of this principle is overcoming centralization. This is simply because the principle makes it impossible for only one party, be it a server, a computer, etc., to have control over the chain. The chain is actually distributed among all its users around the world. Anyone can easily download the chain, have access to it and be part of it. This means more 
safety as hackers cannot tamper with, or hack, the chain unless they hack every single user of it (Iansiti and Lakhani, 2017).

3. Mining process:

This means that millions of computers around the world take part in making sure that a given transaction is correct before it can be completed. If a user wanted to transfer money to another via the blockchain the transaction would not be completed, even if the money was available, unless mining occurred first. Mining means employing the abilities of the computers in searching for the correct "Hash" that distinguishes the transaction so that it can be completed successfully. Using their computers, miners around the world engage in sophisticated mathematical processes with the aim of arriving at the correct hash that links the current transaction to the previous transaction and distinguishes it from other transactions occurring in the chain. Mining is undertaken with a view to making sure that all the transactions entering the chain have taken the same time that the it took before in previous blocks (Kaspersky, 2017).

Once the correct hash is arrived at, the new transaction is permitted into the chain and is added to the other transactions in the blocks, eventually forming a blockchain (bitcoinexchangeguide, 2018). This makes it difficult for hackers to hack the system or even manipulate it, as doing such things would require hacking all the computers at the same time. Once the transaction is verified it can be completed. The miner who arrives at the correct hash gets a percentage of the transferred money, by way of reward. If the matter involves transferring Bitcoin currency the miner gets a reward for taking part in the mining process.

Any blockchain consists of four main elements: the block (which is the unit of which the chain is made), information, hash and timestamp. Following are more details:

- The block: A block is a number of processes or tasks that must be undertaken inside the chain. Examples are money transfer, data registration, following up a case, etc. Usually each block can accommodate a specific number of processes and information. No additional information or processes are accepted. Once the processes in question are completed a new block, related to what has gone before, is created. The main objective here is to avoid any fake processes that can cause the chain to freeze or be inoperative.

- Information: This refers to subsidiary processes that take place inside a given block. In other words, it is a single order that occurs in the block and, together with other orders and information, constitutes the block itself.

- Hash: This can be described as the chain's DNA, occasionally referred to as the digital signature. It is a code produced via an algorithm in the blockchain software, called "Hash function" (Northcutt, 2018).

A hash performs the following functions:

- Distinguishing the chain from other chains: as each chain has a unique hash.

- Distinguishing one block from another: as, again, each block has its own hash.

- Distinguishing each piece of information by means of a unique hash.

- Linking the chain's blocks to one another: as each block is linked to both the preceding hash and the following hash. Thus, the hash moves in one direction only-namely, from the block in question to the one next to it, and so on. This is why a hash renders ineffectual any attempt to make alterations to the already created blocks.

4. Timestamp: Or the time which any process inside the chain has taken (Parker, 2018). 


\section{APPLICATIONS OF BLOCKCHAIN}

In addition to being used in transferring money, both traditionally and virtually, a Blockchain can serve many, different purposes. These will be explained in the following section:

1. Registering property: A Blockchain can be used in registering property, be it personal assets (e.g. real estate, jewelry, precious stones, cars, etc.), patents, or intellectual property rights ( of books, songs, poems, etc.). It can even be used in registering ordinary ideas which cannot be regarded as innovations or accomplishments in the usual sense of the word. In brief, A Blockchain can be used in registering whatever one owns and wishes to advertise or register with the aim of preventing others from claiming it, so that $\mathrm{s} / \mathrm{he}$ can sell it later or use it in transactions via the Blockchain system (Malinger, 2017).

2. Registering transactions: This applies to transactions between individuals, inside companies or inside governmental or non-governmental institutions. This is simply because the Blockchain system allows everyone to insert all kinds of data, whether these data have to do with government transactions (Medium, 2017), production line tracking, planes and tankers routing, financial transactions registering, property transfer, and customer service follow-up, to name but a few of the kinds of transactions that the system can be used for registering. This facilitates detecting gaps and weak points, combating corruption and controlling quality.

3. Facilitating mediation: The Blockchain system replaces traditional intermediary parties. Instead of banks (in money transfer), real estate departments (in registering property), traffic departments (in registering vehicles), brokers (in financial transactions) and mediator service companies (such as Uber), millions of people around the world who use the Blockchain act as mediators, benefiting from the financial outcome which traditional mediators would have got in return for their services. Small as it is, the outcome the Blockchain users achieves equal distribution of wealth.

The processes involved in using the Blockchain system can be clarified via some practical examples, as follows:

In case an individual wishes to buy a piece of land from another, s/he accesses the ledger where all users have registered their assets and engages in the transaction of buying the needed piece of land from its owner. Based on the aforementioned principle of open ledger as it is, the Blockchain system gives the potential buyer access to a detailed history of the piece of land in question, including the exact dates on which it was sold and bought till it came to be owned by its current owner.

Once negotiations end and all is settled, the current owner transfers the ownership of the piece of land to the new owner via the same ledger. Not only will all users witness the transaction but they will actually contribute to its success as verifying it is no longer the responsibility of the real estate department but these users' responsibility. There will be no need to consult the city and neighbourhood services department in charge of information concerning land ownership and transactions, simply because large numbers of miners, even millions of them in some cases, will take part in the verification of the transaction. Though they will be paid for their efforts necessary to guarantee the security of the transaction, what they get is far less than what traditional verification entities get. This enhances the principle of equal distribution of wealth, no matter how small this "wealth" is.

Not only can the Blockchain system play an effective role in transferring the piece of land's ownership, but it can also be utilized in transferring the buyer's money to the seller with no need for banks and like mediators. This can be achieved through depending on the medium using Blockchain, such as Due.com. Depending on such a nontraditional mediator saves both costs and time; while traditional money transfer can take three to seven days, transfer via Blockchain only takes a few minutes and no mediation fees are paid; the user only pays for using the system, in which case the fees are low.

An important question in this connection is: can the ledger be hacked and can the data be tampered with? The answer is: this is very unlikely to happen since the ledger is not a single central database but a database distributed among users around the world. In other words, each user has his/her own copy of the ledger. Consequently, in order for a hacker to hack a transaction occurring in the ledger s/he has to be 
able to hack each and every user of the ledger, on condition that hacking them all should occur simultaneously. This is extremely difficult, if not impossible, to happen, because the hacking attempt, which will naturally take the form of a transaction, will not be completed unless all users confirm and register it.

Blockchain can also be employed in sophisticated administrative processes, such as tracking a container ship travelling from, say, China to Europe but has to stop at a number of ports for such purposes as unloading and loading goods, getting fuel, getting food for the crew, etc. The first step here would be creating a block for the journey-namely, the Genius block. This will contain all the information about the starting point of the journey and the containers that the ship is carrying. The hash mechanism creates the hash that will distinguish this block and connect all the chain's blocks to one another. It will also create the timestamp for performing the transaction in the chain.

Once the ship arrives at the first port, where it will unload some containers before it resumes its journey, a new block will be created in the chain, containing information about the containers which have been unloaded, the hash of the new block and the timestamp. The second hash is henceforth linked to the first hash so that any attempt to make alterations to the information concerning the starting point of the journey will be rejected by the chain.

By the time the ship reaches its final destination a large number of blocks will have been accumulated. Each of these blocks will represent a point where a number of containers were unloaded, and each of these points will be linked to a hash which, in return, will be linked to all the preceding and following hashes. Thus, all hashes will be moving in one direction, from the original block to the last block. Because no alterations are allowed, failure to unload containers, or failure to unload them in the right port, can be easily detected, and the party accountable can be easily identified. In addition, this method prevents stealing and scamming, as each container is registered in the chain and all the details of its route are retraceable. As a result, if a container contained smuggled goods or drugs, the doer, or at least the party that facilitated such a crime through negligence, could be easily identified.

\section{BENEFITS OF USING THE BLOCKCHAIN TECHNOLOGY}

Blockchain offers its users many advantages. As an administrative and financial system, it is capable of fulfilling many purposes, meanwhile saving time, effort and costs. It also makes it possible to monitor all operations and processes and detect their initiators, guaranteeing a high degree of security. Besides, it remarkably reduces the risk of fraud and manipulation, thanks to the hash mechanism, here are the following:

1. Offering an effective alternative to bureaucratic red tape: Using the Blockchain system in government departments means more efficiency, as all information concerning transactions, as well as documents, certificates, etc., are assigned definite positions in the chain, and can be easily retrieved whenever need arises. This helps avoid time-consuming red tape associated with traditional, bureaucratic government transactions (Williams, 2017).

2. Guaranteeing quality assurance: The Blockchain allows users to keep track of the transaction's progress status, which results in high-quality services. For instance, using Blockchain in a food production line guarantees that the final products will be characterized by their remarkable quality (Agrawal, 2018).

3. Combating corruption: The Blockchain is almost immune to fraud and manipulation attempts, simply because it does not allow users to modify or delete transactions. As a matter of fact, the system keeps track of all the steps of transactions, as well as of the exact time each step takes place. In case an attempt of manipulation occurs the system rejects the transaction and prevents its initiator from re-trying. This helps minimize the threat of corruption.

4. Equal distribution of wealth: Using the Blockchain system puts an end to the monopoly certain corporations and organizations hold on wealth, guaranteeing that everyone has a fair share of wealth, since all people around the world can take part in managing transactions and get paid for this. 


\section{POTENTIAL THREATS OF BLOCKCHAIN}

Despite its various advantages, the Blockchain technology can pose certain threats, among the most serious of which are the following:

1. Endangering mediating jobs and institutions: This is by far the most serious threat of the Blockchain system as far as monetary, administrative and intermediary sectors are concerned. Depending on the Blockchain system means that, gradually, the need for such sectors would diminish, and they could eventually be "extinct", so to speak. This situation is similar to the implications of industrialization for certain crafts and professions which are now history because of the many technological advances that have made them dispensable. Unless the aforementioned sectors adapt to the new situation, they will meet a similar destiny (Cardone, 2017).

2. Abuse of the system: The Blockchain system can be used in illegal activities, such as drug dealing, prostitution, arms trafficking, etc. Needless to say, this constitutes a great threat to society's security and individuals' welfare and interests.

3. The possibility of stealing users' personal information: Though Blockchains are immune to manipulation from outside the chain, users in the chain can steal the personal information of other users and use it in manipulating these users' assets, selling them, or even endangering these users' jobs, etc.

4. Endangering service availability and accessibility: This is still a potential threat that can paralyze the chain, despite the fact that the system is designed so as to prevent such a risk through limiting the capacity of each block (Medium, 2017).

5. Hacking: Though, as already mentioned, it is rather difficult to hack Blockchain because this requires that all users and miners be hacked simultaneously, hacking remains a potential threat. This is particularly true for chains with relatively small numbers of users and miners.

\section{CHALLENGES AND STUMBLING BLOCKS}

Perhaps it is worth noting that the Blockchain technology has not gained wide currency and acceptance among people yet. It has not yet invaded many fields where it would be of great use. Following are some of the stumbling blocks facing the Blockchain Technology:

1. Problems that have to do with popularity: Though the Blockchain technology has been in use for more than ten years, it did not gain enough popularity among users. It was not "openly" used for any purposes other than exchanging Bitcoin currency. Therefore, it can be said that the system has not gained wide acceptance yet, the reason probably being the technical sophistication of the system which makes it difficult to use (Kasireddy, 2017).

2. Problems pertaining to replacing the old traditional systems with the new one: These have to do with the stability that the old systems have acquired in the course of time due to the fact that, despite their shortcomings, they have always done the job efficiently. Banks and money transfer companies, registration offices and like institutions have been operating for decades, gaining acceptance, authority and credibility over the years, which makes it difficult for the relatively new Blockchain system to simply take over from them, especially in some sectors.

3. Lack of trust: People may feel less willing to trust the Blockchain system because information about its designer is lacking. All we know is that in 2008, with the world financial crisis, a person supposedly called Satoshi Nakamoto devised a new system for exchanging currency without needing mediation from banks and financial institutions. The system, which has come to be known as Blockchain, has been used ever since for exchanging Bitcoin currency.

4. Issues that have to do with accountability: The aforementioned avoidance of centralization has its own shortcomings. In case faults, hacking or scam occur, it will be impossible to decide who is responsible since there is no one party or entity that can be considered in 
charge of controlling and managing the system and consequently held accountable for the situation.

5. Issues that have to do with cost: doing the needed transactions and making transfers need many computers with certain specifications. Besides, these computers consume a lot of energy, which should be taken into consideration, given the recent considerable increase in energy prices.

\section{REFERENCES}

Agrawal, J. (2018, January 18). 8 Benefits of Blockchain to Industries Beyond Cryptocurrency. Retrieved from https://www.entrepreneur.com/article/306420

An Analysis of the Opportunities and Threats in Blockchain Technology (2018, February 13). Retrieved from https://medium.com/the-mission/an-analysis-of-the-opportunities-and-threats-in-blockchaintechnology-6f55d647be3e

A Scalable Blockchain Database (2016). BigchainDB, Berlin, Germany, June 8, p1.

Betsy, P. (2017, May 31). Blockchain - Distributed Ledger Technology Application Benefits? Retrieved from https://bitcoinexchangeguide.com/blockchain-distributed-ledger-technology/

BlockChain Technology (2015). Sutardja Center for Entrepreneurship \& Technology Technical Report. Brekely University of California, October 16, p1.

BlockChain Technology (2017), Ob cit, p5

Eaton-Cardone, M. (2017, January 4). How blockchain will affect financial services employment. Retrieved from https://news.efinancialcareers.com/gulf-en/269710/how-blockchain-will-affectfinancial-services-employment/

Eyal Malinger, B. (2017, November 18). Blockchain could 'change everything' for real estate. Retrieved from https://venturebeat.com/2017/11/18/blockchain-could-change-everything-for-real-estate/

Iansiti, M., \& Lakhani, K. R. (2017). The Truth About Blockchain. Retrieved from https://hbr.org/2017/01/the-truth-about-blockchain

Kasireddy, P. (2017, December 11). Fundamental challenges with public blockchains. Retrieved from https://medium.com/@preethikasireddy/fundamental-challenges-with-public-blockchains$253 \mathrm{c} 800 \mathrm{e} 9428$

Northcutt, S. (2018). Hash Functions, SANS TM Technology Institute. Retrieved from https://www.sans.edu/cyber-research/security-laboratory/article/hash-functions

Parker, L. (2015, February 10). Timestamping on The Blockchain. Retrieved from https://bravenewcoin.com/news/timestamping-on-the-blockchain/

Six myths about blockchain and Bitcoin: Debunking the effectiveness of the technology (2017, August 18). Kaspersky. Retrieved from https://www.kaspersky.com/blog/bitcoin-blockchainissues/18019/

Tapscott, D. (2016, June). How the blockchain is changing money and Business. TED Summit. Retrieved from

https://www.ted.com/talks/don_tapscott_how_the_blockchain_is_changing_money_and_business

Williams, S. (2017, December 11). 5 Big Advantages of Blockchain, and 1 Reason to Be Very Worried, Fool. Retrieved from https://www.fool.com/investing/2017/12/11/5-big-advantages-ofblockchain-and-1-reason-to-be.aspx 